\title{
Philosophiques
}

Katia Genel (dir.), La dialectique de la raison. Sous bénéfice d'inventaire, Éditions de la Maison des sciences de l'homme, Paris, 2017, 312 pages

\section{Aurélia Peyrical}

Volume 45, numéro 2, automne 2018

URI : https://id.erudit.org/iderudit/1055283ar

DOI : https://doi.org/10.7202/1055283ar

Aller au sommaire du numéro

Éditeur(s)

Société de philosophie du Québec

ISSN

0316-2923 (imprimé)

1492-1391 (numérique)

Découvrir la revue

Citer ce compte rendu

Peyrical, A. (2018). Compte rendu de [Katia Genel (dir.), La dialectique de la raison. Sous bénéfice d'inventaire, Éditions de la Maison des sciences de l'homme, Paris, 2017, 312 pages]. Philosophiques, 45(2), 546-551.

https://doi.org/10.7202/1055283ar d'utilisation que vous pouvez consulter en ligne.

https://apropos.erudit.org/fr/usagers/politique-dutilisation/ 
"corps propre", distingue par le caractère localisé de ses sentiments les bonnes causes des bons effets. L'être angélique, comme un pilote dans son navire, parce qu'il n'a pas ce rapport intime à son corps, ne peut opérer cette discrimination. L'ouvrage se clôt sur une réflexion d'ensemble sur ce que le physicalisme a donc manqué par rapport à Descartes: que le corps sentant (humain) est irréductible aux corps en général. Cette conclusion s'appuie sur un survol des différents problèmes auxquels se butent les positions physicalistes lorsqu'elles traitent des questions épineuses de la conscience phénoménale et des qualia. "L'effet que cela fait " correspondant à tel phénomène physique localisable serait en définitive toujours néanmoins irréductible au physique. Le mérite devant être accordé à Descartes et à son concept d'union serait donc d'avoir pressenti l'irréductibilité d'un vécu primitif humain, inexplicable et inanalysable en sa totalité, laissant sur son passage une empreinte indélébile.

VINCENT DARVEAU-ST-PIERRE

Université de Montréal

École normale supérieure de Lyon

\begin{abstract}
Katia Genel (dir.), La dialectique de la raison. Sous bénéfice d'inventaire, Éditions de la Maison des sciences de l'homme, Paris, 2017, 312 pages
\end{abstract}

Le volume La dialectique de la raison. Sous bénéfice d'inventaire, dirigé par $\mathrm{K}$. Genel, rassemble les contributions du colloque international qui s'est tenu à la Sorbonne les I 2 et I 3 mai 20 I 4 sous le titre "La Dialectique de la Raison, 70 ans après. Critique sociale, politique et esthétique ${ }^{1}{ }^{\star}$. L'ouvrage ne rend pas précisément compte du colloque tel qu'annoncé, puisque les contributions de J.-O. Bégot, A. Birnbaum et M. E. Rosen ne s'y trouvent pas. S'y ajoutent en revanche celles d'A. Allen, A. Grivaux et E. Buch. Aux quatre moments du colloque ${ }^{2}$ se substituent trois temps dans l'ouvrage publié: «Un modèle critique inédit », "Faire table rase et après? Le modèle critique de la Dialectique de la Raison en question", "Au-delà de la critique: un autre discours rationnel». Le choix d'une telle réorganisation ne nous paraît pas anodin. À un ensemble de thèmes - métaphilosophie, éthique et esthétique, sciences sociales, politique - est préféré un arrangement qui, en décloisonnant les questionnements, propose un cheminement progressif centré sur la question de l'actualité de ce texte phare de la seconde moitié du vingtième siècle. $\mathrm{K}$. Genel souligne en introduction que l'intention

1. https://calenda.org/ 285898

2. I. La forme de la critique: écriture philosophique et critique de la société; II. Théorie critique, éthique et esthétique; III. Modèles critiques: sciences sociales et philosophie de l'histoire; IV. Les enjeux politiques de la Dialectique de la Raison. 
d'une telle publication «est de restituer [au texte] ses ambivalences et de montrer que le diagnostic historique sur les Lumières s'articule à un discours sur l'usage même de la raison qui a gardé toute sa pertinence» (p. Io).

L'enjeu correspond à l'idée que l'on se fait d'une théorie critique vivante, confrontée à des défis aussi inédits que multiples: ne pas clore définitivement les interprétations d'un texte complexe et à maints égards étonnant, ne pas prétendre parvenir à en aplanir les tensions multiples. Les contributions, dans leur diversité, semblent animées d'une même quête: dépasser la perplexité première sans la sous-estimer, afin d'aller au-delà tant des polémiques stériles les plus variées que d'une révérence hors de propos. $\mathrm{Ni}$ document historique ni monument intouchable, donc. Afin d'éviter ces écueils, les contributrices et contributeurs s'efforcent, par des réflexions elles-mêmes dialectiques, de susciter la (re)lecture de cet ouvrage difficile, alors même que certains ont voulu le discréditer en y voyant une "sorte d'autodestruction de la critique sous sa forme totalisante» (p. Io).

Les contributions de la première partie brossent le portrait d'un texte qui n'en finit pas de surprendre. Sans aller jusqu'à assimiler la raison communicationnelle de Habermas à l'Aufklärung totalitaire décriée par Adorno et Horkheimer, A. Allen cherche à évaluer le dépassement promis par ce dernier. Elle propose de revisiter la Dialectique de la Raison pour y voir une source d'inspiration possible pour les développements actuels de la théorie critique, notamment les critiques postcoloniales. Son argument consiste à voir dans l'ouvrage, dans sa rhétorique et son style, non pas la volonté de résoudre le paradoxe de l'Aufklärung, mais le souci pérenne de présenter l'ambivalence de la raison sous forme polémique et incisive, d'en exposer ainsi la foncière ambiguïté.

A. Demirovic s'interroge également sur la pertinence de la critique habermassienne lorsqu'elle discrédite l'entreprise de la Dialectique de la Raison au nom d'un échec à refonder la raison et ses prétentions à l'universel. Comme il le souligne à plusieurs reprises, pour les auteurs «[i]l n'est nullement besoin de refonder la raison, car celle-ci, dans la vie collective, ne cesse d'être exercée » (p. 93). Chercher un programme d'émancipation dans la Dialectique de la Raison revient à miminiser sa stratégie originale: analyser, à même l'exercice historiquement différencié de l'Aufklärung, le fossé entre ses tendances irrationnelles, qui résulte de son affinité avec la domination, et son indéniable parenté avec l'émancipation. Demirovic ne le dit pas explicitement, mais cet argument est crucial pour éviter une lecture des théoriciens de Francfort, par exemple de Dialectique négative, comme étant des apologies de l'immédiateté, voire de la misologie.

À rebours de la lecture de la Dialectique de la Raison comme un texte unifié, J. Abromeit rend sensible la tension entre deux concepts d'Aufklärung. Il oppose ainsi l'historicisme critique de Horkheimer à l'historisme généalogique et anthropologisant (p. 67) d'Adorno, le second ayant finalement pris le dessus dans l'ouvrage, bien que des traces du premier soit encore pré- 
sentes. Il accorde personnellement ses sympathies au premier type d'historisme, plus concret et différencié selon lui, et invite les recherches actuelles à se ressaisir d'un tel concept. Partisan de l'historicisme, il termine son article de façon très laconique sur un rapprochement selon lui possible entre la tendance anthropologique et l'ontologie existentiale de Martin Heidegger, sans toutefois faire référence aux textes spécifiques d'Adorno ou de Horkheimer. Il n'évoque pas non plus, à notre grande surprise, le volume de contributions en langue anglaise qui instruit ce parallèle 3 . Nous avons exprimé nos propres réticences concernant ce rapprochement à l'occasion de la parution du dernier ouvrage de P. Gordon ${ }^{4}$.

O. Voirol renvoie dos à dos deux interprétations admises de la Dialectique de la Raison. Pour ce qui est de la lecture pessimiste, le livre entérine la résignation des penseurs face à l'essoufflement du projet initial de critique de la science, ne voyant plus dans la philosophie bourgeoise des Lumières de quoi porter l'émancipation. Pour la seconde, la Dialectique de la Raison ne peut être que faire figure d'une fable littéraire stimulante mais philosophiquement pauvre, trop peu en prise avec les contenus scientifiques de son temps, trop jargonnante. Si cependant on replace la Dialectique de la Raison dans son contexte, cette double lecture est-elle pertinente? Contrairement à J. Abromeit qui proposait une lecture interne des tensions propres au texte, Voirol conclut à la force critique de l'unité d'exposition de la Dialectique de la Raison comme "mode d'exploration échappant aux catégories de l'abstraction et de la pensée de l'identité »(p. 84). Celui-ci, loin d'être un frein aux recherches en sciences sociales, vient les interroger dans leur épistémologie implicite.

Les articles de M. Gangl et K. Genel se complètent dans la mesure où ils abordent tous deux la façon dont le fascisme allemand fut pensé par Adorno et Horkheimer. M. Mangl parvient à la conclusion que la version définitive du texte représente moins un tournant "anthropologique " qu'elle ne fait fond sur la théorie du capitalisme d'État de Pollock. De même, K. Genel rappelle que l'effacement du vocabulaire marxiste se fait au profit d'un vocabulaire explicitement lié aux débats théoriques sur l'autoritarisme dans les années 30 vis-à-vis desquels la Dialectique de la Raison se positionne. L'auteure fait alors le point sur le rapport d'Adorno et Horkheimer aux concepts de totalitarisme et d'autoritarisme. L'originalité du livre consiste à penser l'autoritarisme et l'autorité comme faits civilisationnels à l'œuvre jusque dans la démocratie, en désignant, au-delà de la structure de l'État, un type d'individualité. La thèse selon laquelle l'Aufklärung est tota-

3. Iain Macdonald, Krzysztof Ziarek (dir.), Adorno and Heidegger: Philosophical Questions, Stanford University Press, 2007.

4. Peter Gordon, Adorno and Existence, Harvard University Press, 20I6. Pour une recension critique de ce texte, nous nous permettons de renvoyer à notre texte sur le site "Actu Philosophia » à l'adresse http://www.actu-philosophia.com/Peter-Gordon-Adorno-and-Existence/ 
litaire - même en la figure de son plus éminent critique, Hegel — dépasse largement le cadre du débat entre politologues. La distinction entre totalitarisme et autoritarisme dans l'ouvrage «tente de circonscrire le dépassement de l'autorité dans le système " social en son entier (p. I35), d'où la critique de ce que nous appellerions volontiers un «individu administré».

La seconde partie de l'ouvrage rassemble des contributions interrogeant la portée de la radicalité propre à la Dialectique de la Raison. A. Grivaux questionne l'influence de la psychanalyse comme discours à même de justifier la thèse centrale de l'ouvrage: l'autodestruction de la raison. Dans la même perspective, G. Raulet analyse en détail la lecture proposée par les auteurs du personnage d'Ulysse dans l'Odyssée, figure-sujet dont les ambivalences reflètent structurellement celles de l'Aufklärung. En développant, selon Raulet, une interprétation résolument anti-marxienne et anti-luckacienne d'Ulysse comme premier bourgeois, Adorno et Horkheimer montrent que le «jeu rusé de la raison avec l'irrationalité ne fait que révéler l'irrationalité réelle de la raison» (p. I64). Le discours psychanalytique, qui irrigue le texte de façon souterraine et lancinante, sert selon A. Grivaux une stratégie délibérément ambiguë: à la fois discours éclairé et discours mythique, cette stratégie permet de désigner l'irrationnel, la déraison, sans tomber dans le discours de l'Aufklärung. On se demande néanmoins ce qui donne à ce discours un tel privilège, qui semble, du moins ici, le placer au-delà d'une dialectique pourtant posée comme générale entre la raison et le mythe. Les deux articles indiquent deux manières dont les auteurs lèvent l'apparence des Lumières comme mouvement de progression sans mélange, alors qu'elles mettent en réalité en place tous les éléments d'un terrorisme de l'intelligibilité.

Le texte anti-habermassien de M. Abensour répond aux soupçons de nihilisme jetés sur la Dialectique de la Raison en proposant d'instruire la distinction entre progressisme et radicalisme. Par une comparaison avec la critique des Lumières opérée par Rousseau, M. Abensour propose de voir dans l'ouvrage une "amplification sans précédent du soupçon que formulait déjà Rousseau à l'égard des Lumières» (p. I76). Le refus du progressisme optimiste n'apparaît plus comme une condamnation univoque des Lumières, mais comme un appel à retrouver ce qui en elle fait signe vers l'utopie.

Par une comparaison cette fois avec la pensée de Derrida, l'étude de C. Menke éclaire la Dialectique de la Raison dans son ambition de penser le monde moderne comme préhistoire. La relation qu'instaure l'Aufklärung entre raison et action ne dépasse en effet pas ce stade. Pour le montrer, Menke procède à une lecture conjointe du différer derridien et de l'inégalité adornienne. Les deux pensées coïncident dans la mise au jour d'une scission dans le sujet moderne. Face à son action, le sujet se trouve pris au piège entre l'idéal qu'il poursuit et l'intelligibilité de son action. L'idéal mythique aussi bien que l'intention d'intelligibilité de la raison calculante, tous deux mus par une logique d'identification, manquent in fine le supplément qui carac- 
térise l'action, et, ce faisant, le Bien lui-même. Pour Adorno comme pour Derrida, en effet, la logique du récit mythique et celle de la ratiocination se distinguent sans différer, car il ne suffit pas que la première prenne la seconde comme repoussoir pour s'en libérer. Si, comme le rappelle Menke, la différence fondamentale entre Adorno et Derrida tient au social (p. 208), on déplorera que l'auteur interprète trop rapidement l'échec de l'Aufklärung comme une simple retombée dans la nature.

Partant d'une comparaison entre les procédés rhétoriques de la Dialectique de la Raison et ceux de la dystopie de G. Orwell, 1984, le texte d'A. Gayraud clôt cette partie de l'ouvrage en posant les jalons d'une lecture ambitieuse de celui-ci comme parodie dystopique des discours de l'Aufklärung. L'originalité d'Adorno et de Horkheimer tient, selon l'auteure, à ce qu'ils ne répondent pas à la philosophie utopiste par une dystopie littéraire qui, malgré son efficace potentielle, lui reste extérieure. Bien plutôt, les premiers théoriciens critiques accusent l'Aufklärung en s'y inscrivant, par le truchement d'un texte qui joue avec les frontières des genres, se faisant ainsi inassignable.

Les quatre dernières contributions du volume envisagent les modalités d'un autre discours rationnel, avec et après la Dialectique de la Raison. S'y croisent activité mimétique, langage réflexif, musique et cri - de jouissance ou d'effroi. Dans son essai, P.-F. Noppen explore la thèse contre-intuitive défendue par Adorno et Horkheimer de l'essence mimétique de la rationalité et de la centralité du mimétisme pour la formation du moi, inaperçue par ce moi lui-même. L'originalité des auteurs par rapport à Hegel tient à ce que la projection n'est en rien un processus strictement naturel mais est médiatisée par une société d'autant plus pathogène qu'elle empêche la constitution des conditions de possibilité de la saisie réflexive, par le moi, de sa constitution mimétique. Dans sa contribution, M. Nobre soutient que l'abandon relatif de la critique de l'économie politique, induit par la Dialectique de la Raison, ne vient pas tant de ce qu'elle formulerait une nouvelle anthropologie que d'une nouvelle manière de procéder au diagnostic du temps présent. La relance du travail interdisciplinaire et des études sociologiques est induite, et non mise à l'arrêt, par la dimension philosophico-anthropologique du texte.

Anne Boissière propose quant à elle une traversée originale dans la Dialectique de la Raison en réinterprétant le rapport d'Ulysse aux Sirènes à l'aune de la Philosophie de la nouvelle musique. La position d'Ulysse, à la fois esclave et maître du rythme saccadé de ses rameurs, manifeste l'échec de la substomption de la pulsion - la danse, à laquelle correspond l'écoute «rythmico-spatiale» — sous la raison — le chant, qui requiert une écoute "expressivo-dynamique». La dialectisation de cette opposition se fait au bénéfice d'une compréhension plus nette de ce à quoi pourrait ressembler l'émancipation. "L'expérience musicale permet de découvrir un rapport de passivité au monde et un laisser-aller dans l'écoute qui n'existe pas autrement» (p. 279), mais elle est en même temps une ruse de la raison, car elle 
requiert une maîtrise technique hautement artificielle. Dans son ambiguité, elle fait signe vers la distinction subtile mais cruciale entre envoûtement et enchantement. Là où l'envoûtement nous coupe des émotions et du corps par une déréalisation hallucinée, l'enchantement alimente le rêve d'une autre modalité d'action que le mouvement mécanique, que la reproduction du monde à l'identique.

En proposant une relecture des événements du I 3 novembre 2015 à Paris, le dernier essai du volume tente une actualisation de la dialectique du son, tout à la fois promesse de jouissance et signe annonciateur de la terreur. E. Buch se demande ainsi ce qui, des rythmes rock, des hurlements des armes ou des sirènes des véhicules d'État, représente une issue hors du monde administré. Toutes, en réalité, sont ambigües, comme l'étaient naguère le chant des sirènes pour Ulysse: chant de mort et chant de jouissance, présence mythique à l'intérieur de la raison qu'elle échoue à conjurer par ses propres moyens, parce qu'elle se croit radicalement autre que lui.

Pluriel et riche, ce volume, on l'a vu, manifeste la grande diversité de voies possibles pour une théorie critique contemporaine soucieuse de sa tradition, dont l'un des jalons, et non des moindres, reste ce conte moderne qu'est la Dialectique de la Raison. Par la multiplicité des interprétations qu'ils suscitent, les textes d'Adorno et de Horkheimer nous offrent encore des armes pour comprendre notre présent.

AURÉLIA PEYRICAL

Université Paris-Nanterre

\section{Marie Garrau, Politiques de la vulnérabilité, Paris, CNRS Éditions, 2018, 358 pages}

Dans son ouvrage "Politiques de la vulnérabilité », la philosophe Marie Garrau présente un projet politique clair qui prend acte de la vulnérabilité fondamentale des sujets et qui valorise une forme d'autonomie relationnelle telle que celles qui sont mises en avant dans l'éthique du care de Joan Tronto et dans la théorie de la reconnaissance d'Axel Honneth. Ainsi, Garrau souhaite montrer que seule une société qui garantit à ses citoyennes et citoyens - à travers une politique de la vulnérabilité - l'accès aux conditions relationnelles et sociales de leur autonomie peut être dite «juste» (Garrau, 20I 8, I7).

L'autrice nous propose, pour ce faire, une analyse de travaux variés portant sur la vulnérabilité. L'aspect pluridisciplinaire de cette démarche est l'un des éléments qui rend cet ouvrage particulièrement pertinent, puisqu'en faisant dialoguer des travaux de philosophie morale et politique ainsi que des travaux de sociologie, Garrau parvient à présenter les éthiques du care non pas comme un courant féministe évoluant parallèlement aux débats 